\title{
(อ) OPEN ACCESS \\ Association of education level with diabetes prevalence in Latin American cities and its modification by city social environment
}

\author{
Ariela Braverman-Bronstein 다, ${ }^{1}$ Philipp Hessel, ${ }^{2}$ Catalina González-Uribe ${ }^{3}$ \\ Maria F Kroker, ${ }^{4}$ Francisco Diez-Canseco, ${ }^{5}$ Brent Langellier, ${ }^{1}$ Diego I Lucumi, ${ }^{2}$ \\ Lorena Rodríguez Osiac, ${ }^{6}$ Andrés Trotta 지, ${ }^{7}$ Ana V Diez Roux ${ }^{1}$
}

\begin{abstract}
- Additional material is published online only. To view, please visit the journal online (http://dx.doi.org/10.1136/ jech-2020-216116).

'Urban Health Collaborative, Drexel University Dornsife School of Public Health, Philadelphia, PA, USA ${ }^{2}$ School of Government, Universidad de los Andes, Bogota, Colombia

${ }^{3}$ School of Medicine, University of Los Andes, Bogota, Colombia ${ }^{4}$ INCAP Research Center for the Prevention of Chronic Diseases, Institute of Nutrition of Central America and Panama, Guatemala City, Guatemala ${ }^{5}$ CRONICAS, Center of Excellence in Chronic Diseases, University Peruana Cayetano Heredia, Lima, Peru

${ }^{6}$ School of Medicine, University of Chile, Santiago, Chile ${ }^{7}$ Institute of Collective Health, National University of Lanus, Buenos Aires, Argentina
\end{abstract}

Correspondence to Ariela Braverman-Bronstein, Urban Health Collaborative, Drexel University Dornsife School of Public Health, Philadelphia, PA 19104, USA; ab4257@drexel.edu

Received 23 November 2020 Revised 14 January 2021 Accepted 23 January 2021 Published Online First 4 February 2021

\begin{abstract}
Background Diabetes prevalence continues to increase in urban areas of low-income and middle-income countries (LMIC). Evidence from high-income countries suggests an inverse association between educational attainment and diabetes, but research in LMIC is limited. We investigated educational differences in diabetes prevalence across 232 Latin American (LA) cities, and the extent to which these inequities vary across countries/ cities and are modified by city socioeconomic factors. Methods Using harmonised health survey and census data for 110498 city dwellers from eight LA countries, we estimated the association between education and diabetes. We considered effect modification by city Social Environment Index (SEI) as a proxy for city-level development using multilevel models, considering heterogeneity by sex and country.
\end{abstract}

Results In women, there was an inverse dose-response relationship between education and diabetes (OR: 0.80 per level increase in education, $95 \% \mathrm{Cl} 0.75$ to 0.85 ), consistent across countries and not modified by SEl. In men, Argentina, Brazil, Colombia, Chile and Mexico showed an inverse association (pooled OR: 0.92; 95\% Cl 0.86 to 0.99). Peru, Panama and El Salvador showed a positive relationship (pooled OR 1.24; $95 \%$ Cl 1.04 to 1.49). For men, these associations were further modified by city-SEl: in countries with an inverse association, it became stronger as city-SEl increased. In countries where the association was positive, it became weaker as citySEl increased.

Conclusion Social inequities in diabetes inequalities increase as cities develop. To achieve non-communicable disease-related sustainable development goals in LMIC, there is an urgent need to develop policies aimed at reducing these educational inequities.

\section{INTRODUCTION}

According to the $\mathrm{WHO}$, diabetes prevalence has doubled over the past 30 years reaching $8.4 \%$ in 2014 , with $75 \%$ of the cases occurring in lowincome and middle-income countries (LMIC). Although diabetes risk factors such as body mass index appear to be increasing in both rural and urban areas, ${ }^{2}$ in many LMIC urban living may be especially conducive to diabetes through living and working environments; promoting sedentary work, processed foods consumption, and automobile transportation, all of which may impact risk factors for diabetes. $^{3-5}$

In 2012, there were 62 million people with diabetes in Latin America and the Caribbean (LAC), this number is predicted to increase to 109 million by $2040 .^{6}$ The LAC region is highly urbanised, with a substantial proportion of persons with diabetes residing in cities. ${ }^{7}$ Evidence from highincome countries (HIC) suggests that diabetes is strongly linked to socioeconomic disadvantage ${ }^{8}$ but studies of the social patterning of diabetes in large and growing cities of LMIC remain scant. ${ }^{9}$ Some evidence suggests that social gradients in diabetes are larger in more urbanised LMIC or in more urbanised regions within LMIC. ${ }^{10-12}$

Several factors may contribute to diabetes inequities in urban areas. Urban living (and changes in income and work associated with it) has been linked to more processed foods consumption, and higher prevalence of drinking, smoking and sedentary lifestyles. $^{9}{ }^{13}$ This increase initially occurs among the wealthiest populations but later transitions into a disproportionate increase in disadvantaged populations. $^{14-17}$

Despite the importance of diabetes in urban areas and the documented presence of stronger diabetes inequities in more urbanised areas, ${ }^{9-11}$ no prior research has examined how specific features of urban areas affect the social patterning of diabetes in cities. Similarly, to what has been observed for countries, cities that have higher incomes, better infrastructure (including housing), and higher proportion of people with higher education levels may also paradoxically exhibit greater educational inequalities in chronic disease risk, ${ }^{18}{ }^{19}$ because beneficial resources and environments may be unequally distributed. Understanding and documenting educational inequities in diabetes in LMIC and its variations across different urban environments is necessary to develop effective interventions to reduce the prevalence of diabetes across rapidly growing cities of LMIC.

Using a harmonised dataset of individual and city-level data for 232 cities in eight Latin American countries, we investigated educational differences in diabetes prevalence by gender, whether these inequities vary across countries and cities, and the extent to which they are modified by city social environment. 


\section{METHODS}

Data are from the Salud Urbana en America Latina Project project, which has compiled and harmonised health, social and built-environment data from 371 large cities (population $\geq 100000$ in 2010) from 11 countries. ${ }^{20}$ These analyses are based on a 232 cities subset from 8 countries with available survey data on diabetes: Argentina, Brazil, Chile, Colombia, El Salvador, Mexico, Peru and Panama. Surveys were designed for chronic disease surveillance using random probabilistic household sampling. Sample sizes for countries ranged from 1333 to 30425 participants. Survey details are provided in online supplemental table 1.

The main outcome was self-reported diabetes in adults 25 years or older. Survey participants were classified as having diabetes if they reported being given a diabetes diagnosis by a healthcare provider. Female participants who reported a diagnosis of gestational diabetes were not considered to have diabetes, except in the case of Argentina and Panama because information on pregnancy status during the time of diagnosis was unavailable.

The two key exposures investigated were city Social Environment Index (SEI) and individual-level education, a proxy of individual-level SES.

The SEI combines four variables representing various aspects of the city social environment: water access (\% households with access to piped water); sanitation (\% households with access to a municipal sewage network); overcrowding (\% households with $>3$ people per room); and primary education completion ( $\%$ population $\geq 25$ years with at least completed primary education). This measure was created by summing the standardised Z-scores of the four variables (overcrowding was reverse coded). The SEI is used as a global metric of city social environment since it captures various aspects of city-living conditions. It has been shown to be related to differences in life expectancy across cities in the sample. ${ }^{18}$ For descriptive analyses, the entire sample was divided into SEI tertiles (low, medium, high).

Individual-level education was obtained from survey reports. Data were harmonised using the Integrated Public Use Microdata Series ${ }^{21}$ harmonisation definitions and categorised into the following categories: (1) less than primary: individuals with no education or less than completed primary education; (2) primary: individuals who completed primary education, but with incomplete secondary education; (3) secondary: individuals with complete secondary education, complete non-university postsecondary education (eg, technical school), or with incomplete university education; (4) university or higher: individuals who completed a university degree or with complete/incomplete graduate studies.

Because descriptive analyses suggested a linear pattern in the association between education categories and diabetes, education was examined as a continuous variable ranging from 0 to 3 reflecting increasing education categories. Age (range 25-97 years) and sex were included as covariates.

\section{Statistical analysis}

Descriptive statistics for all predictors are presented by city-SEI tertiles and diabetes status. We estimated age-adjusted proportions by education categories and gender separately for each country using Poisson regression models. We inspected patterns (ie, whether they were approximately graded or exhibited thresholds) as well as major differences across countries in the nature of the association (eg, inverse, none, positive).
We estimated the association of city-SEI and individuallevel education with diabetes using two-level mixed logistic regression models stratified by sex with individuals nested within cities, adjusting for country fixed effects. We included a random intercept for each city and a random slope for education to allow education gradients to differ between cities. Model 1 included the two key predictors city-SEI and individual-level education, as well as age. Model 2 included country fixed effects. In model 3, we included interactions between education and country fixed effects to determine if there were differences in education gradients by country. If the interaction was statistically significant, we further examined heterogeneity by grouping countries into two groups based on educational patterns observed in descriptive analyses (group 1, displaying an inverse crude association: Argentina, Brazil, Chile, Colombia, Mexico; group 2, displaying a positive crude association: Peru, Panama, El Salvador) and including an appropriate interaction term.

To test our hypothesis that city-SEI modified educational differences, we fitted model 4 which added an interaction term between city-SEI and individual-level education (both as continuous variables). Using this model, we estimated the effect of a one-level increase in education for people living in cities at the 10th, 50th and at the 90th percentiles of city-SEI. We plotted the predicted probabilities resulting from the model according to education level by city-SEI.

All analyses were stratified by sex considering previous evidence suggesting that the effects of social environment and education on non-communicable diseases (NCDs) vary by sex. ${ }^{22}$ For the models, city-SEI was standardised to a mean of 0 and a SD of 1 . We specified a level of significance of 0.05 and all analysis was conducted using SAS 9.4 and R V.3.6.1 software.

\section{RESULTS}

Our sample included 110498 survey participants in 232 cities from eight Latin American countries. There was a median of 300 individuals per city (IQR 120-600). Table 1 shows city and individual-level characteristics according to city-SEI. The full sample had a median age of 43 years and 59\% were women. The highest percentage of survey participants lived in Brazil and Mexico (27.5\% and 21.2\%, respectively), while El Salvador and Chile had the lowest $(1.2 \%$ and 2.2\%, respectively). Age and gender did not differ across SEI tertiles. The percentage of people with diabetes was 7\%,9\% and $8 \%$, respectively, at the lowest, middle and highest city-SEI tertile. Higher SEI cities tended to have a larger proportion of inhabitants within higher educational categories. Persons with diabetes lived in cities with a slightly higher SEI were older and more concentrated in the lower education categories than those without diabetes (online supplemental table 2).

Figure 1 shows the age-adjusted proportion of respondents with diabetes by education level by sex. In women, there was a clear inverse association between diabetes and education for all countries except Peru; additionally, in Argentina, Brazil and Mexico there was a dose-response pattern. In men, there was an inverse association in Argentina, Brazil and Chile without a consistent dose-response relationship, but no clear pattern was observed in Mexico and Colombia, although a slightly higher prevalence of diabetes was observed in the lower educational categories. In men living in Peru, Panama and El Salvador, there was a positive association with a doseresponse pattern: the higher the education level, the higher the diabetes prevalence. 
Table 1 City-level and individual-level characteristics by city-level SEI tertile

\begin{tabular}{|c|c|c|c|c|c|}
\hline & Overall & Low SEI & Medium SEI & High SEI & $P$ value \\
\hline \multicolumn{6}{|l|}{ City characteristics } \\
\hline Number of cities & 232 & 98 & 64 & 70 & \\
\hline City-SEl & $0.28(-0.14,0.46)$ & $-0.36(-0.76,-0.14)$ & $0.28(0.18,0.39)$ & $0.58(0.46,0.76)$ & $<0.001$ \\
\hline \multicolumn{6}{|l|}{ SEI components } \\
\hline$\%$ households with piped water & $89.9(81.2,95.0)$ & $77.8(74.8,87.0)$ & $90.8(85.6,95.3)$ & $94.2(90.3,95.5)$ & $<0.001$ \\
\hline$\%$ households with sewage network & $78.3(54.7,88.0)$ & $52.7(29.5,78.3)$ & $75.6(56.7,88.0)$ & $87.2(80.1,94.4)$ & $<0.001$ \\
\hline$\%$ households with overcrowding & $3.4(2.1,6.0)$ & $6.8(3.7,10.5)$ & $3.9(2.4,5.3)$ & $2.7(1.7,3.1)$ & $<0.001$ \\
\hline$\%$ population 25 and older with at least primary education & $79(72.9,84.3)$ & $75.2(69.7,82.3)$ & $78.3(72.7,81.6)$ & $82.9(76.3,87.3)$ & $<0.001$ \\
\hline \multicolumn{6}{|l|}{ Individual-level characteristics } \\
\hline Number of survey respondents & 110498 & 37213 & 34939 & 38346 & \\
\hline Age & $43(34,56)$ & $42(33,55)$ & $44(35,58)$ & $44(34,57)$ & $<0.001$ \\
\hline Women \% & 58.9 & 59.7 & 58.0 & 58.9 & $<0.001$ \\
\hline Diabetes \% & 8.0 & 7.0 & 9.0 & 8.1 & $<0.001$ \\
\hline \multicolumn{6}{|l|}{ Education } \\
\hline Less than primary $\%$ & 19.3 & 22.7 & 18.5 & 16.8 & $<0.001$ \\
\hline Primary \% & 33.2 & 28.3 & 34.2 & 37.1 & \\
\hline Secondary \% & 32.9 & 36.6 & 30.8 & 31.3 & \\
\hline University \% & 14.6 & 12.5 & 16.6 & 14.8 & \\
\hline \multicolumn{6}{|l|}{ Survey respondents per country } \\
\hline Argentina \% & 16.7 & 4.0 & 34.2 & 13.2 & $<0.001$ \\
\hline Brazil \% & 27.5 & 31.3 & 29.7 & 22.0 & \\
\hline Chile $\%$ & 2.2 & 0 & 0.1 & 6.2 & \\
\hline Colombia \% & 13.8 & 8.8 & 12.6 & 19.7 & \\
\hline Mexico \% & 21.2 & 18.5 & 22.8 & 22.3 & \\
\hline Panama \% & 8.4 & 7.6 & 0 & 69.3 & \\
\hline Peru \% & 9.1 & 26.2 & 0.8 & 0 & \\
\hline El Salvador \% & 1.2 & 3.6 & 0 & 0 & \\
\hline
\end{tabular}

We present median, 25 th and 75 th percentiles unless specified otherwise. P values were estimated using Kruskal-Wallis test for continuous variables and $\chi^{2}$ test for discrete variables. Citylevel SEl: Standardised sum of \% of households with piped water, \% of households with sewage network, \%of household with overcrowding (reversed), and \% of population 25 and older with at least primary education.

Given the patterns observed in figure 1, we tested for multiplicative interactions between education and country. The global test for education-country interaction was statistically significant in men $(p=0.005)$ but not in women $(p=0.4)$. We also tested for heterogeneity across sets of countries with different patterns based on visual inspection of figure 1 . In women, we found no evidence that associations differed significantly in Peru ( $p$ for interaction $=0.8$ ) or Chile, Colombia, Panama and El Salvador $(\mathrm{p}=0.2)$ compared with Argentina, Brazil and Mexico. Consequently, we estimated associations of education with diabetes pooling across all countries but adjusting for country fixed effects. In men, we found that associations differed significantly in Peru, El Salvador and Panama compared with the other countries ( $p$ for interaction=0.002). Hence, we provide two estimates of education associations: one for Colombia, Mexico, Argentina, Brazil and Chile, and another for Peru, El Salvador and Panama.

Table 2 shows the ORs of diabetes associated with individuallevel education and city-SEI. In women, higher individual-level education was strongly and inversely associated with lower odds of diabetes, after adjusting for country fixed effects (OR $0.80 ; 95 \% \mathrm{CI} 0.75$ to 0.85$)$. No associations were observed for city-SEI. The random effects for the intercept and education slope remained significant even after adjusting for country, providing evidence of residual variation in diabetes and the effect of education across cities.
In men, after adjusting for country fixed effects higher individual-level education was associated with lower odds of diabetes in Argentina, Brazil, Chile, Colombia and Mexico (0R 0.92; 95\% CI 0.86 to 0.98). For Peru, Panama and El Salvador, the association was reversed, the odds of diabetes increased by $24 \%$ per one-unit higher education level (OR 1.24; 95\% CI 1.04 to 1.49 ), after adjusting for city-SEI, age and country fixed effects. City-SEI was not associated with diabetes. The random intercept remained significant suggesting of residual variability in diabetes between cities. The variance of the random slope for education was no longer statistically significant when we introduced the interaction between education and country.

Table 3 shows associations of individual-level education with diabetes for cities with different levels of SEI. In women, there was no evidence of SEI-education interaction $(p=0.834)$ and the odds of diabetes were reduced by $20 \%$ for each one-unit increase in education level across the different SEI levels. The random slope for education remained statistically significant suggesting residual variation in the effect of education across cities.

In men, there was a statistically significant interaction $(p=0.019)$ between education and city-SEI. We present the OR of diabetes associated with education separately for the two sets of countries given qualitative differences in the directions of the associations between education and diabetes. Men in Argentina, Brazil, Chile, Colombia and Mexico had an inverse association 
Age adjusted percentage of women with diabetes by education level
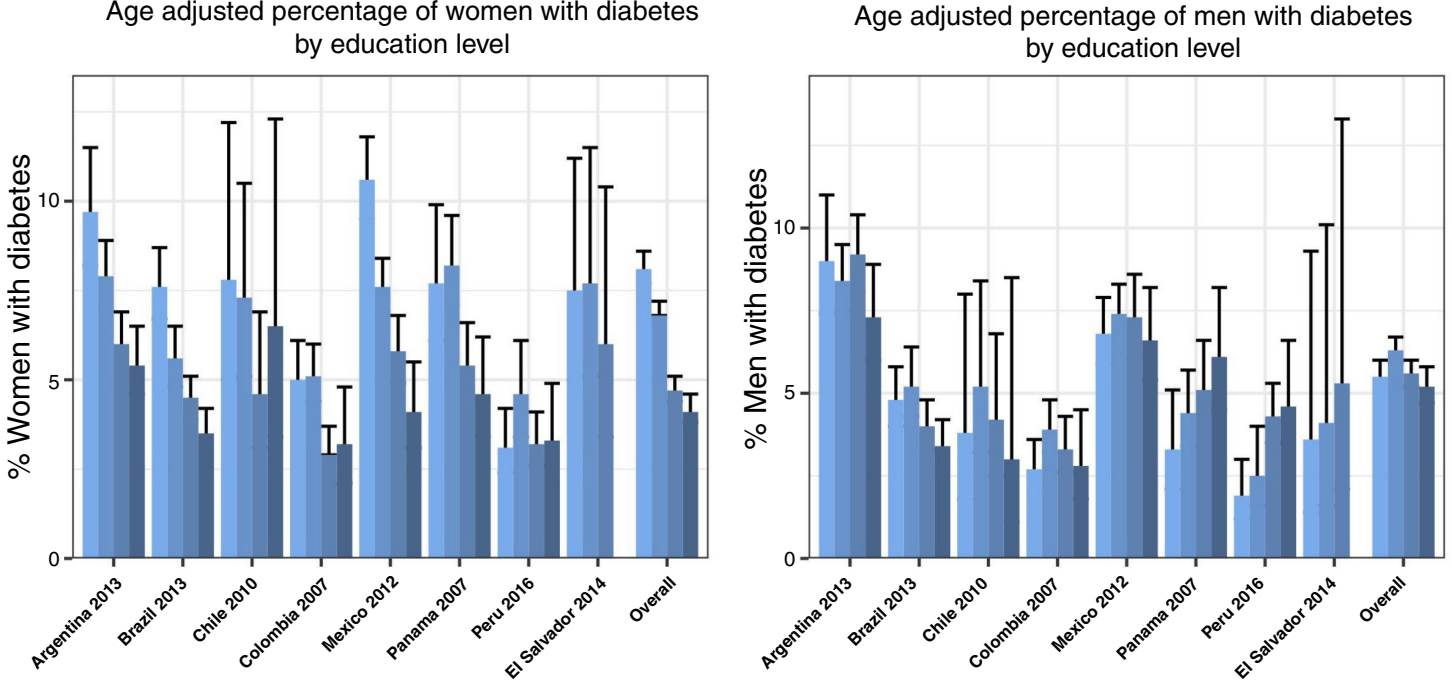
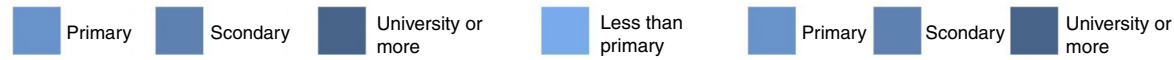

Figure 1 Age-adjusted percentage of people with diabetes by country and gender according to their individual education level. Age was categorised into three groups: 25-39, 40-64, and 65 and older. These categories were further included in Poisson regression models to estimate diabetes prevalence by education levels.

of education with diabetes at higher levels of SEI but it reversed and became non-significant at lower levels (OR (95\% CI) 1.03 (0.92 to 1.16$) ; 0.90$ (0.85 to 0.97$)$ and 0.85 (0.78 to 0.93$)$ for the 10th, 50th and 90th percentiles of SEI, respectively). For men in Peru, Panama and El Salvador, the positive association of education with diabetes weakened and became non-significant as city-SEI increased (OR (95\% CI): 1.33 (1.10 to 1.61$) ; 1.17$ (0.97 to 1.41$)$ and $1.09(0.89$ to 1.35$)$ for the 10 th, 50 th and 90 th percentiles, respectively). The variance of the random slope for education was reduced compared with the model without the
SEI-education interaction and was not statistically significant. Figure 2 depicts these patterns graphically for predicted probabilities derived from the model with interactions.

\section{DISCUSSION}

We used survey data for 232 large cities in eight Latin American countries to characterise educational differences in diabetes prevalence and examine the extent to which these inequities varied across countries and cities. We also examined whether city

Table 2 ORs of diabetes associated with individual-level education and city-SEl by sex

\begin{tabular}{|c|c|c|c|}
\hline & Model 1 & Model $2 \dagger$ & Model 3‡ \\
\hline Individual and city characteristics* & OR $(95 \% \mathrm{Cl})$ & OR $(95 \% \mathrm{Cl})$ & OR $(95 \% \mathrm{Cl})$ \\
\hline \multicolumn{4}{|l|}{ Women $(n=51903)$} \\
\hline City-SEI & $1.00(0.95$ to 1.06$)$ & $0.96(0.91$ to 1.02$)$ & - \\
\hline Education level & $0.78(0.73$ to 0.83$)$ & $0.80(0.75$ to 0.85$)$ & - \\
\hline \multicolumn{4}{|l|}{ Random effects } \\
\hline Intercept variance (SE) & $0.0798(0.0197)$ & $0.0364(0.0135)$ & - \\
\hline Education slope variance (SE) & $0.0262(0.0155)$ & $0.0259(0.0142)$ & - \\
\hline \multicolumn{4}{|l|}{ Men $(n=37246)$} \\
\hline City-SEI & 1.02 (0.96 to 1.09$)$ & 0.97 (0.92 to 1.03$)$ & $0.98(0.92$ to 1.05$)$ \\
\hline Education level & $0.93(0.87$ to 1.00$)$ & 0.95 (0.89 to 1.02$)$ & \\
\hline Argentina, Brazil, Chile, Colombia, Mexico & - & - & $0.92(0.86$ to 0.99$)$ \\
\hline Peru, Panama, El Salvador & - & - & $1.24(1.04$ to 1.49$)$ \\
\hline \multicolumn{4}{|l|}{ Random effects } \\
\hline Intercept variance (SE) & $0.1004(0.0240)$ & $0.0201(0.0117)$ & $0.0210(0.0117)$ \\
\hline Education slope variance (SD) & $0.0346(0.0168)$ & $0.0209(0.0127)$ & $0.0163(0.0124)$ \\
\hline
\end{tabular}

Bold values have a $\mathrm{p}$ value $<0.05$.

*All models were adjusted for individual age. Education is modelled as a continuous variable, a one-unit increase reflects one higher level of education, for example, less than primary to primary complete. City Social Environment Index (SEI) was standardised to a mean of 0 and an SD of 1 . The OR is estimated for a 1 SD (0.58703) difference in SEI.

tModel 2 is adjusted for country fixed effects.

¥Model 3 includes an interaction between education and different country groups only for men ( $p$ for interaction $=0.002$ ). Combinations of the main effect of education and the interaction coefficient were used to derive estimates for different countries. 
Table 3 ORs $(95 \% \mathrm{CI})$ of diabetes associated with education for cities with different levels of the Social Environment Index (SEI) and residual education slope variance across cities

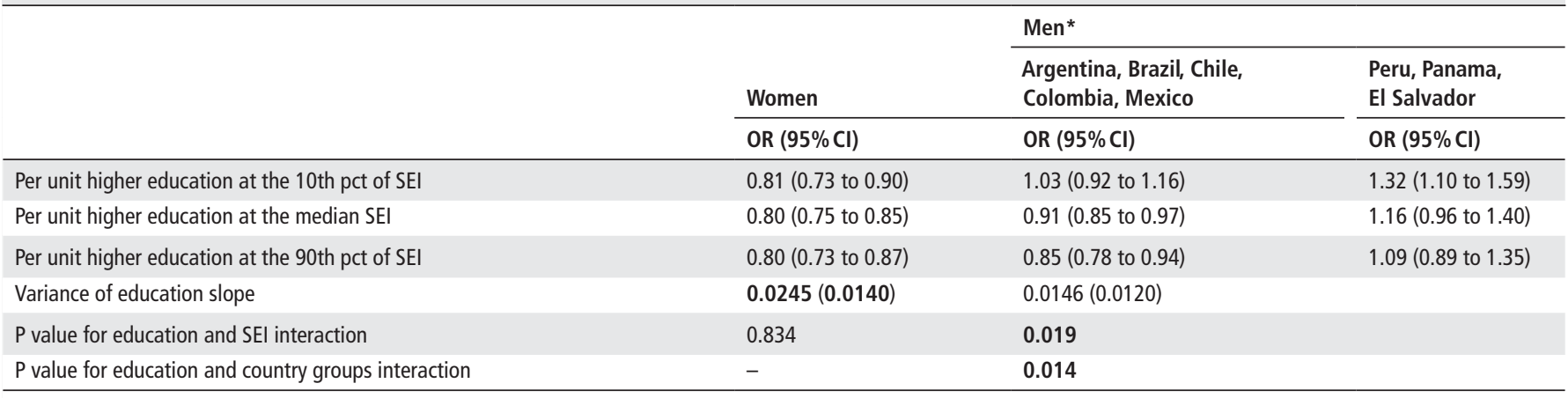

Models were adjusted for age, country fixed effects, and education and SEI main effects.

* The model for men also includes the interaction between individual education and country.

social environment modified the relationship between education and diabetes. In women, we found a clear educational gradient; women with higher education levels had a lower proportion of diabetes, with a dose-response pattern for all countries (except possibly Peru). Furthermore, this association was not modified by city-SEI. In men, the association of education with diabetes varied across countries; in some countries, there was an inverse association while in other countries there was no association or the opposite association (higher proportion of diabetes in men with higher education). Moreover, this association was modified by city-SEI such that an inverse association emerged (or a positive association weakened) as the city-SEI improved.

Evidence from HIC consistently shows a higher prevalence of diabetes in people with lower education levels compared with higher education and this association is consistently stronger in women. ${ }^{22-24}$ Limited data from LMIC suggest heterogeneity in social gradients. ${ }^{12} 25-27$ We found that among Latin American women in urban areas, the association of education with diabetes follows the same pattern found in HIC. ${ }^{22}$ For men, the pattern is not constant across countries. In the case of Peru, El Salvador and Panama, we found a higher prevalence of diabetes among men with higher education.

Other research from HIC and some Latin American countries has documented stronger inverse educational or socioeconomic gradients in obesity, diabetes, and NCDs in women but weaker or opposite gradients in men. ${ }^{12} 1622232527-29$ Education may be more beneficial to women because they may lack other resources such as earnings, power and authority. ${ }^{30}$ Social norms regarding weight, physical activity and diet (all risk factors for diabetes) maybe more strongly patterned by education in women than in men resulting in differential associations of diabetes with education by sex. ${ }^{28}$

In our study, country modified educational gradients in men but not in women. The countries in which we observed that higher education was associated with more diabetes in menincluding Peru and El Salvador-are the countries with the lowest income per capita in our sample. ${ }^{31}$ These findings are consistent with prior work showing that country economic development modifies social gradients in chronic disease risk. ${ }^{425}$ In the early stages of the nutritional transition, men with higher socioeconomic status tend to be less physically active and consume more processed foods with high content of fat and salt, compared with men in lower socioeconomic status. As countries develop, this pattern reverses and those with higher income and education have healthier diets and behaviours. ${ }^{9} 173233$ Why this modification of educational gradients by country was more evident in men than in women is a question that deserves additional research.

Our sample includes 232 cities from eight Latin American countries, with large heterogeneity in social environments.
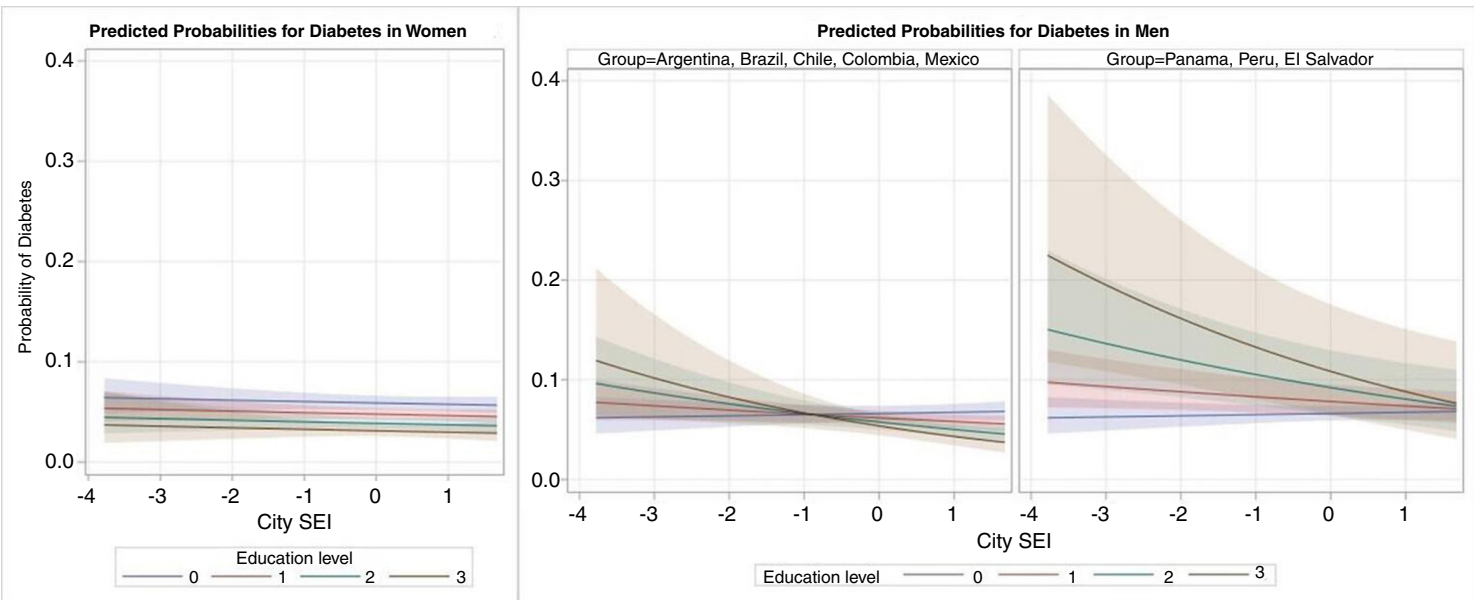

Figure 2 Predicted probabilities of diabetes based on the multilevel logistic regression models to assess the effect modification of education level by city-Social Environment Index (SEI). Education level: $0=$ =ess than primary; $1=$ primary; $2=$ secondary; $3=$ university or more. City-SEI values are based on the standardised variable used in the model so the range of values differs from the on presented in table 1. 
Thus, we expected our results to vary between cities. No effect modification was observed in women. In men, we found that city-SEI modified the association of education with diabetes. In countries with an inverse association, it became significant and was stronger as city-SEI increased. In countries where the association was positive, it became weaker as city-SEI increased. These results mimic the country-level differences but across cities within a country. These findings are consistent with a recent study in India which found that the positive association between diabetes and individual SES in less developed districts became weaker or inverse in districts with higher levels of development. ${ }^{26}$ Our results highlight the importance of considering the different social and economic environments within a region and how the effects of individual-level factors may be modified by environmental conditions.

An important limitation of our study is the use of self-report survey data for the ascertainment of diabetes status. This may have led to underestimates in groups with less access to healthcare and may have consequently resulted in underestimates of educational gradients. Gender differences in access to healthcare, such as prenatal care identification of high-risk women, and pattering of healthcare access by SES could also have impacted the gender differences that we observed. The year of the surveys and census measures are not fully aligned across countries, although some stability in the census-based measures of population characteristics can be assumed. ${ }^{3}$ Because our focus was on estimating associations rather that prevalences for specific

\section{What is already known on this subject}

- Previous evidence from high-income countries reports an inverse association of diabetes with individual-level socioeconomic status; however, studies of the social patterning of diabetes in low-income and middle-income countries (LMIC) are scant. Some evidence suggests that social gradients in diabetes are larger in LMIC that are more urbanised compared with less urbanised ones and that social gradients also vary within regions of LMICs, with larger social gradients observed in more compared with less urbanised areas. However, no studies have examined inequities in a systematic fashion across the growing cities of LMIC or how these gradients may vary across countries or be modified by city characteristics.

\section{What this study adds}

- This is the first study to comprehensively examine social gradients in diabetes across the cities of Latin America. We found that in women there was a clear educational gradient in diabetes: women with higher education levels had a lower proportion of diabetes, with a dose-response pattern for all countries. Furthermore, this association was not modified by city social environment. In men, the association of education with diabetes varied across countries and this association was modified by city social environment as such that an inverse association emerged (or a positive association disappeared) as the city socioeconomic conditions improved. It is paramount to develop policies aimed at reducing social inequities in diabetes which can be expected to increase over time as cities continue to grow and develop unequally. cities we did not use survey weights; however, variables related to the weights were included as adjustment factors. Our results are cross sectional and cannot be used to draw causal inferences but still provide valuable descriptive information relevant to policy. Major strengths of our study include the large sample of individuals (110 498) and cities (232) representing a significant proportion of the urban population of Latin America, the harmonised dataset, and the availability of city-specific information absent from past work. Our models are adjusted for country fixed effects that would account for any unmeasured country factors such as differences in healthcare and education systems across countries.

In summary, we found strong educational gradients in diabetes in women living in Latin American cities, regardless of city socioeconomic development. Findings in men suggest that inverse gradients may emerge as country and city socioeconomic development increase. Our results show that social gradients varied and modified by country and local contexts. Considering the increasing trends of diabetes and the high urbanisation in Latin America, it is paramount to develop policies aimed at reducing social inequities in diabetes in cities. These policies need to be sensitive to the ways in which inequities in diabetes manifest in different social and economic contexts and how they may vary across countries and cities. Targeting socioeconomic and gender inequities in cities will be critical to achieving the sustainable development goals related to reducing NCDs in LMIC.

Correction notice This article has been corrected since it first published. The 7th affiliation has been corrected.

Twitter Ariela Braverman-Bronstein @aribravs

Acknowledgements The authors acknowledge the contribution of all SALURBAL project team members. For more information on SALURBAL and to see a full list of investigators, see https://drexel.edu/lac/salurbal/team/. SALURBAL acknowledges the contributions of many different agencies in generating, processing, facilitating access to data or assisting with other aspects of the project. Visit https://drexel.edu/lac/ data-evidence for a complete list of data sources.

Contributors PH and AVDR conceived the study. AB-B carried out the statistical analyses. $A B-B$ and AVDR drafted the first version of the manuscript. AT, MFK and FD-C participated in or supported data collection and revised the manuscript. CG-U, $B L$, DIL and LRO revised and edited the manuscript, as well as supporting literature search. All authors participated in the interpretation of the results, reviewed and approved the final version of the manuscript.

Funding The Salud Urbana en América Latina (SALURBAL)/ Urban Health in Latin America project is funded by the Wellcome Trust (205177/Z/16/Z).

Competing interests None declared.

Patient consent for publication Not required.

Ethics approval The SALURBAL study protocol was approved by the Drexel University IRB (ID\#1612005035) and by appropriate site-specific IRBs.

Provenance and peer review Not commissioned; externally peer reviewed.

Data availability statement Data are available upon reasonable request. The SALURBAL project welcomes queries from anyone interested in learning more about its dataset and potential access to data. To learn more about SALURBAL's dataset, visit https://drexel.edu/lac/ or contact the project at salurbal@drexel.edu.

Supplemental material This content has been supplied by the author(s) It has not been vetted by BMJ Publishing Group Limited (BMJ) and may not have been peer-reviewed. Any opinions or recommendations discussed are solely those of the author(s) and are not endorsed by BMJ. BMJ disclaims all liability and responsibility arising from any reliance placed on the content. Where the content includes any translated material, BMJ does not warrant the accuracy and reliability of the translations (including but not limited to local regulations, clinical guidelines, terminology, drug names and drug dosages), and is not responsible for any error and/or omissions arising from translation and adaptation or otherwise.

Open access This is an open access article distributed in accordance with the Creative Commons Attribution 4.0 Unported (CC BY 4.0) license, which permits others to copy, redistribute, remix, transform and build upon this work for any purpose, provided the original work is properly cited, a link to the licence is given, 
and indication of whether changes were made. See: https://creativecommons.org/ licenses/by/4.0/

ORCID iDs

Ariela Braverman-Bronstein http://orcid.org/0000-0002-9999-808X

Andrés Trotta http://orcid.org/0000-0003-4181-1494

\section{REFERENCES}

1 World Health Organization (WHO). Diabetes, 2020. Available: https://www.who.int/ news-room/fact-sheets/detail/diabetes [Accessed 30 Jul 2020].

2 NCD Risk Factor Collaboration (NCD-RisC). Rising rural body-mass index is the main driver of the global obesity epidemic in adults. Nature 2019:569:260-4.

3 Jaacks LM, Vandevijvere S, Pan A, et al. The obesity transition: stages of the global epidemic. Lancet Diabetes Endocrinol 2019;7:231-40.

4 Aschner P, Aguilar-Salinas C, Aguirre L, et al. Diabetes in South and Central America: an update. Diabetes Res Clin Pract 2014;103:238-43.

5 Miranda JJ, Barrientos-Gutiérrez T, Corvalan C, et al. Understanding the rise of cardiometabolic diseases in low- and middle-income countries. Nat Med 2019:25:1667-79.

6 Ogurtsova K, da Rocha Fernandes JD, Huang Y, et al. IDF diabetes atlas: global estimates for the prevalence of diabetes for 2015 and 2040. Diabetes Res Clin Pract 2017;128:40-50.

7 Gwynne RN. Industrialization and urbanization in Latin America. Routledge, 2017

$8 \mathrm{Xu} Z$, Yu D, Yin X, et al. Socioeconomic status is associated with global diabetes prevalence. Oncotarget 2017;8:44434-9.

9 Allen L, Williams J, Townsend N, et al. Socioeconomic status and non-communicable disease behavioural risk factors in low-income and lower-middle-income countries: a systematic review. Lancet Glob Health 2017;5:e277-89.

10 Gassasse Z, Smith D, Finer S, et al. Association between urbanisation and type 2 diabetes: an ecological study. BMJ Glob Health 2017;2:e000473.

11 Fleischer NL, Diez Roux AV, Alazraqui M, et al. Socioeconomic gradients in chronic disease risk factors in middle-income countries: evidence of effect modification by urbanicity in Argentina. Am J Public Health 2011;101:294-301.

12 Perez Ferrer C, McMunn A, Rivera Dommarco JA, et al. Educational inequalities in obesity among Mexican women: time-trends from 1988 to 2012. PLoS One 2014;9:e90195

13 Stringhini S, Bovet P. Socioeconomic status and risk factors for non-communicable diseases in low-income and lower-middle-income countries. Lancet Glob Health 2017;5:e230-1.

14 Gómez-Dantés H, Fullman N, Lamadrid-Figueroa $\mathrm{H}$, et al. Dissonant health transition in the states of Mexico, 1990-2013: a systematic analysis for the global burden of disease study 2013. Lancet 2016:388:2386-402.

15 Pérez-Ferrer C, McMunn A, Zaninotto P, et al. The nutrition transition in Mexico 1988-2016: the role of wealth in the social patterning of obesity by education. Public Health Nutr 2018;21:2394-401.
16 Monteiro CA, Conde WL, Popkin BM. Independent effects of income and education on the risk of obesity in the Brazilian adult population 1. American Society for Nutritinal Sciences, 2001: 881S-6. https://academic.oup.com/jn/article/131/3/881S/4687033

17 Baker P, Machado P, Santos T, et al. Ultra-processed foods and the nutrition transition: global, regional and national trends, food systems transformations and political economy drivers. Obes Rev 2020;21:e13126.

18 Bilal U, Alazraqui M, Caiaffa WT, et al. Inequalities in life expectancy in six large Latin American cities from the SALURBAL study: an ecological analysis. Lancet Planet Health 2019;3:e503-10.

19 Lê-Scherban F, Ballester L, Castro JC, et al. Identifying neighborhood characteristics associated with diabetes and hypertension control in an urban AfricanAmerican population using geo-linked electronic health records. Prev Med Rep 2019:15:100953.

20 Quistberg DA, Diez Roux AV, Bilal U, et al. Building a data platform for Cross-Country urban health studies: the SALURBAL study. J Urban Health 2019:96:311-37.

21 IPUMS International. Available: https://international.ipums.org/international/ [Accessed 24 Aug 2020].

22 Backholer K, Peters SAE, Bots SH, et al. Sex differences in the relationship between socioeconomic status and cardiovascular disease: a systematic review and metaanalysis. J Epidemiol Community Health 2017;71:550-7.

23 Fleischer NL, Henderson AK, Wu Y-H, et al. Disparities in diabetes by education and Race/Ethnicity in the U.S., 1973-2012. Am J Prev Med 2016;51:947-57.

24 Espelt A, Borrell C, Roskam AJ, et al. Socioeconomic inequalities in diabetes mellitus across Europe at the beginning of the 21st century. Diabetologia 2008:51:1971-9.

25 Seiglie JA, Marcus M-E, Ebert C, et al. Diabetes prevalence and its relationship with education, wealth, and BMI in 29 low- and middle-income countries. Diabetes Care 2020;43:767-75.

26 Jung L, De Neve J-W, Chen S, et al. The interaction between district-level development and individual-level socioeconomic gradients of cardiovascular disease risk factors in India: a cross-sectional study of 2.4 million adults. Soc Sci Med 2019;239:112514.

27 Williams J, Allen L, Wickramasinghe K, et al. A systematic review of associations between non-communicable diseases and socioeconomic status within low- and lower-middle-income countries. J Glob Health 2018;8.

28 Tang M, Chen Y, Krewski D. Gender-related differences in the association between socioeconomic status and self-reported diabetes. Int J Epidemiol 2003:32:381-5.

29 Kautzky-Willer A, Dorner T, Jensby A, et al. Women show a closer association between educational level and hypertension or diabetes mellitus than males: a secondary analysis from the Austrian his. BMC Public Health 2012;12:1-9.

30 Ross CE, Mirowsky J. Gender and the health benefits of education. Sociol $Q$ 2010;51:1-19.

31 World bank open data | Data. Available: https://data.worldbank.org/ [Accessed 18 Aug 2020].

32 Popkin BM, Reardon T. Obesity and the food system transformation in Latin America. Obes Rev 2018;19:1028-64.

33 Pou S, Tumas N, Aballay L. Nutrition Transition and Obesity Trends in Argentina Within the Latin American Context. In: Faintuch J, Faintch S, eds. Obesity and diabetes. Cham: Springer, 2020 